Recepción: 12/09 / 2018

Aceptación: $10 / 11 / 2018$

Publicación: 20 / 01/ 2019

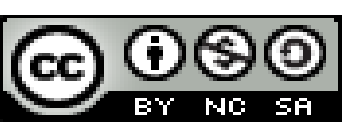

Ciencias económicas y empresariales

\title{
El Marketing Digital un pilar fundamental en la gestión estratégica financiera de la industria turística
}

\section{Digital Marketing a fundamental pillar in the strategic financial management of the tourism industry}

\section{Marketing Digital é um pilar fundamental na gestão financeira estratégica da indústria do turismo}

Juan P. Mancheno-Saá I

juanpa-007@hotmail.com

\section{Christian A. Barragán-Ramírez II \\ cbarragan@pucesa.edu.ec}

\section{Sonia F. Chaluisa-Chaluisa ${ }^{\mathrm{III}}$ soniach2@gmail.com \\ Alicia G. Ortiz-Morales ${ }^{\mathrm{IV}}$ gioviortiz@hotmail.com}

\section{Correspondencia: juanpa-007@hotmail.com}

\footnotetext{
${ }^{\text {I }}$ Máster Universitario en Dirección y Administración de Empresas, Ingeniero Comercial con Mención en Marketing, Docente Universidad Técnica de Ambato, Ambato, Ecuador.

II Magíster en Administración de Empresas Mención Planeación, Ingeniero Comercial con Mención en Marketing, Docente Universidad Pontificia Universidad Católica del Ecuador de Ambato, Ambato, Ecuador.

III Magíster en Gestión de Empresas Mención Pequeñas y Medianas Empresas, Ingeniera Comercial, Docente Universidad Técnica de Ambato, Ambato, Ecuador.

IV Magíster en Gerencia Financiera Empresarial, Magíster en Pedagogía y Gestión Educativa, Licenciada en Contabilidad y Auditoría Contadora Publica, Doctora en Contabilidad y Auditoría, Docente Universidad Técnica de Ambato, Ambato, Ecuador.
} 


\title{
Resumen
}

El marketing digital es la nueva percepción en el mundo actual, indicando que este empieza a ser un puntual fundamental en el desarrollo empresarial, el consumidor que por el vínculo web ha sido denominado internauta cada vez es más especializado, haciendo que la UX o User Experience de las páginas web evolucionen mucho más cuando se trata de industrias gráficas y multimedia. Una caracterización de primer y segundo nivel en la industria turística permitirá identificar los factores de éxito tanto en la gestión física como en la digital, creando una realidad mixta denominada blended. La metodología utilizada es de naturaleza descriptiva con dos fuentes específicas de conocimiento:

- La investigación bibliográfica, la cual hace referencia a los autores y obras más representativas dentro de publish or perish

- Investigación de Campo con métodos cualitativos y cuantitativos ejecutada en el año 2018

La caracterización encontrada facilita a que los dueños de hoteles, hosterías y hostales tengan presente varias de las características del turista promedio, por otra parte, sirve de una fotografía al sector tomando como referencia un sector representativo de la economía ecuatoriana, segmentado en la provincia. El presente estudio pretende ser un parámetro más no un estudio de caso específico con una realidad específica de un hotel en particular.

Palabras claves: Industria hotelera; mercado turístico; turista; hotel; caracterización turística

\begin{abstract}
Digital marketing is the new perception in the world today; indicating that this is beginning to be a fundamental point in business development, the consumer that has been known as the web link is becoming more specialized, making the UX or User Experience of web pages evolve much more when it comes to graphic and multimedia industries. A characterization of first and second level in the tourism industry will identify the success factors in both physical and digital management, creating a mixed reality called blended. The methodology used is descriptive in nature with two specific sources of knowledge:
\end{abstract}


- Bibliographic research, which refers to the most representative authors and works within publish or perish

- Field research with qualitative and quantitative methods executed in 2018

The characterization found makes it easier for the owners of hotels, hostels and hostels to keep in mind several of the characteristics of the average tourist; on the other hand, it serves as a photograph of the sector taking as reference a representative sector of the Ecuadorian economy, segmented in the province. The present study aims to be a parameter rather than a specific case study with a specific reality of a particular hotel.

Keywords: Hotel industry; tourist market; tourist, hotel; tourist characterization

\section{Resumo}

Marketing digital é a nova percepção no mundo de hoje, indicando que isso está se tornando um ponto-chave no desenvolvimento de negócios, o consumidor de que o link da web tem sido chamado internauta é cada vez mais especializada, tornando UX ou User Experience de páginas da web evoluem muito mais quando se trata de indústrias gráficas e multimídia. Uma caracterização do primeiro e do segundo nível na indústria do turismo identificará os fatores de sucesso na gestão física e digital, criando uma realidade mista denominada blended. A metodologia utilizada é de natureza descritiva com duas fontes específicas de conhecimento:

- Pesquisa bibliográfica, que se refere aos autores mais representativos e trabalha dentro de publicar ou perecer

- Pesquisa de campo com métodos qualitativos e quantitativos executados em 2018

Facilita a caracterização constatou que os proprietários de hotéis, pousadas e albergues são apresentam várias características do turista médio, por outro lado, serve a indústria de uma fotografia por referência a um setor representativo da economia equatoriana, segmentado na província. O presente estudo pretende ser um parâmetro e não um estudo de caso específico com uma realidade específica de um hotel em particular.

Palavras-chave: Hotelaria; mercado turístico; turista; hotel; caracterização turística 


\section{Introducción}

El marketing digital es una de las herramientas contemporáneas que ha cambiado el mundo y su percepción, el consumidor actual tiene todas las características de un nativo tecnológico, haciendo que la percepción del mismo este enfocada netamente a medios digitales, creando una realidad subjetiva o complementaria. (Chaffey, y otros, 2012)

El Marketing es una ciencia que avanza a pasos agigantados dejando en el pasado el tradicionalismo, también conocido como marketing 1.0 para dar paso al marketing bidireccional que está basado en la inmediatez de la respuesta y en la micro segmentación. (Erragcha, y otros, 2014)

Los mercados tradicionales de diversa naturaleza empiezan a llegar a su fin, como efecto de la globalización donde los productos o servicios son cada vez más especializados, los que nunca dejarían de lado un perfil que tenga una capacidad adquisitiva considerable. El estudio de un producto a través de la investigación de mercados se ha estandarizado en un plazo de aproximadamente cinco años antes de ver la luz en el mercado, esto con el fin de conocer tendencias y macro tendencias que se arraigan en los consumidores. (Kurzwell, 2000). El mercado cambia y las empresas deben responder a estos cambios para lo cual las relaciones bidireccionales se potencian, el medio digital parecería mandar sobre el medio físico creando una percepción virtual que parece permanecer en el tiempo. (Burgos, 2009)

El marketing digital no solo ha servido para vender percepciones, soluciones e incitar al deseo, sino que por otra parte ha servido para recolectar, analizar y gestionar datos, convirtiéndose en una herramienta fundamental en la personalización de productos y mejoramiento continuo de los mismos. (Cangas Muxica \& Guzmán Pinto, 2010).

El objetivo del Marketing digital es similar al tradicional, con una ventaja que este primero tiene una estructura de costos que, con el tiempo, permitirá reflejar beneficios e índices de posicionamiento a los cuales antes hubiera sido imposible llegar (Iglesias, 2013).

El Marketing digital en la actualidad se ha convertido en medio de información 24/7 ya que las personas a través de dispositivos móviles pueden acceder a la misma en cualquier momento independientemente del entorno. (González, 2011). El término marketing digital parece ser muy 
nuevo contemplado en un entorno modernista, sin embargo, este también ha sufrido cambios e innovación, ha pasado de ser un término estático para convertirse en uno de los términos más dinámicos del planeta. Las concepciones del mismo son diferentes de acuerdo al territorio en el que se manejan en USA se le denomina Marketing Online, en la mayoría de países de Europa se le conoce como Marketing en la Web y el resto del mundo lo ha bautizado como Marketing Digital. (Equipo Vértice, 2007)

La personalización de la tecnología es algo muy cercano al nuevo marketing digital la UX o User Experience como se lo conoce es la satisfacción que tiene el internauta al momento de interactuar con un elemento web, (Rowland, y otros, 2015) de aquí que los sitios aparte de ser prácticos y funcionales deben tener una capacidad de encender los sentidos del internauta y motivar a ciertas acciones determinadas. (Sevilla, 2008)

Los fundamentos del marketing digital parecen estar definidos explícitamente, para poder explicar cuál es la secuencia lógica del actuar en la web se deberían gestionar varias técnicas como el Analizador Web, El posicionamiento SEM - referido al marketing en buscadores, el emailing, el posicionamiento SEO o posicionamiento en redes sociales, la optimización de links también llamado link buildings .(Brodkin, 2015). (González, y otros, 2015)

Los principales sitios web en el mundo suelen considerar la influencia directa e indirecta de la información online sobre el mercado potencial y real, donde se pretende cuadrar la satisfacción y fidelidad del cliente. (Hanson, y otros, 2000). El marketing digital ha trasformado la forma de llegar al consumidor, y el consumidor ha trasformado con su llegada al marketing digital para siempre. (Núñez, 2010)

La ventaja infinita del marketing digital es su naturaleza y alcance, pensar que hoy en día puede ser más efectivo un tweet que un anunció en televisión, algo que parecía impensable o imposible, (Gutiérrez, 2008). Existen algunas referencias del tema a nivel contemporáneo: 
Tabla 1

\begin{tabular}{|c|c|c|c|c|}
\hline Autor & Año & Definición & Libro & Citas \\
\hline $\begin{array}{l}\text { Kates, } \\
\text { Matthew }\end{array}$ & 2013 & $\begin{array}{l}\text { "la construcción de conciencia y } \\
\text { promoción de una marca usando } \\
\text { todos los canales digitales } \\
\text { disponibles: Web, SEM (que } \\
\text { incluye el SEO y el sistema de } \\
\text { publicidad de pago por click), } \\
\text { smartphones, mercados móviles } \\
\text { (Google Play, Apple Store), } \\
\text { marketing por email, banners } \\
\text { publicitarios online y social } \\
\text { media". }\end{array}$ & $\begin{array}{l}\text { Making digital } \\
\text { and traditional } \\
\text { marketing } \\
\text { work together }\end{array}$ & $\begin{array}{c}\text { (Matthew, } \\
\text { 2013) }\end{array}$ \\
\hline $\begin{array}{c}\text { Brinkley, } \\
\text { Claire }\end{array}$ & 2012 & $\begin{array}{l}\text { "la promoción de productos o } \\
\text { marcas mediante varias vías de } \\
\text { medios electrónicos. Los medios } \\
\text { que pueden ser usados como } \\
\text { parte de una estrategia de } \\
\text { mercadotecnia digital de un } \\
\text { negocio puede incluir esfuerzos } \\
\text { de promoción vía internet, social } \\
\text { media, teléfonos móviles, } \\
\text { billboards electrónicos y } \\
\text { también mediante la televisión y } \\
\text { la radio". }\end{array}$ & $\begin{array}{l}\text { Marketing } \\
\text { Digital }\end{array}$ & $\begin{array}{c}\text { (Brinkley, } \\
\text { 2012) }\end{array}$ \\
\hline
\end{tabular}

"término que refiere a diferentes técnicas promocionales enfocadas a alcanzar clientes mediante vías tecnológicas. El marketing digital tiene una Clark, 2012 extensa selección de servicios, Dorie 2012 productos y técnicas de

Digital Marketing

(Clark, 2012) mercadotecnia para la marca, que generalmente usan el internet como el principal medio promocional, en adición a la TV y la radio tracional".

"Al marketing digital también se

Philip le conoce como "marketing de

Kotler internet" pero sus procesos

Marketing Internacional

(Kotler, 2013) actuales difieren, ya que el digital está considerado como 
más orientado a ciertos sectores,

más medible y con más

posibilidades de ser interactivo".

\section{Fuente: Elaboración Propia}

El posicionamiento web es una herramienta que con el tiempo pasó del aspecto técnico militar o hasta espacial para aterrizar en campos comunes como la banca, el comercio y hasta a nivel social con las redes, es indispensable indicar que una de las industrias que más se ha visto favorecida por los avances tecnológicos es la de turismo, donde vender una percepción visual ha hecho que la demanda se potencie de forma exponencial. (Ryan, 2016)

La micro segmentación a nivel mundial cambio de una segmentación absolutista, a una micro segmentación diferenciada con varios factores, que con el tiempo daría origen al término de marketing y al comportamiento del consumidor. La aparición de dispositivos móviles, aplicaciones y los refuerzos de percepción generados por el 24/7 de la web ha complicado mucho más una realidad que en un principio fue una isla con respecto al mercado real y hoy en día ha llegado a representar el mismo en su mayoría. (Lozano, y otros, 1999)

La relación entre marketing, ventas y una realidad web ha hecho que la interrelación de términos aumente hasta el punto de que la interacción se ha denominado como un embudo direccionado a la conversión de todo el proceso. (Pérez, 2001)

La web ha dejado de ser una ventaja competitiva y ha empezado a ser un requisito que con el pasar del tiempo puede hacer que una empresa pierda mucho si no la tiene, pero no gane casi nada al manejarla por una saturación comercial digital. (De Vicuña, 2018)

De esta manera los mercados han pasado a ser nichos específicos a través de segmentación profunda y especifica naciendo así el término de targetting del consumidor. (Moschini, 2012)

El marketing en esta nueva etapa ha potenciado uno de sus objetivos más trascendentales, el estudio del mercado y del consumidor que cada día se vuelve más impredecible, las cookies, los historiales de navegación y el registro de compras permiten establecer un perfil indispensable en el actuar de una empresa actual. (Cangas Muxica \& Guzmán Pinto, 2010).

El marketing Digital es aquel que intenta obtener réditos con la menor inversión posible apalancándose en las herramientas del marketing digital. (Adolpho, 2011) 
Las redes sociales horizontales y verticales son una nueva forma de llegar al cliente; muchas veces los empresarios piensan que estos son los principales medios para poder comercializar un producto, pero también es necesario contar con un sitio Web y el apoyo de e-mail marketing, para poder realizar una sinergia digital de medios que permite captar los huecos existentes propios de cada uno de los medios o canales utilizados. (Pablo Segovia, 2011).

El marketing holístico necesariamente debe considerar al marketing digital y la gestión del mismo, entendiendo que "la satisfacción de los clientes está íntimamente ligada con la calidad, la calidad afecta directamente el desempeño de los productos y, por tanto, la satisfacción de los clientes". (Medina, 2011)

El marketing 2.0 unidireccional sin impacto de la retroalimentación ha muerto, por lo que la naturaleza del marketing digital es la concepción del futuro, y brinda un entorno para el desarrollo integro de la ciencia (MarcadorDePosición1) (Chakravorti, 2010). La nueva competencia en el siglo veinte y uno hace referencia a como se aborda un cliente y como se lo fideliza. (Consoli, y otros, 2010)

El marketing tradicional, la logística comercial y las estrategias deben adaptarse a la nueva realidad para tener alcance en el nuevo entorno del consumidor. El marketing digital es más que tener una empresa o página en internet, es aprovechar los medios para que el alcance publicitario sea mayor. (Tuten, 2008)

La segunda parte del marketing digital es cumplir con todo lo prometido y mostrado en la web para poder acceder a una posición privilegiada a nivel mental (Ugarte Otero, 2011)

La idea de mantener seguidores referenciales llamados cookies, es ver el comportamiento del internauta en tiempo real para de acuerdo a esto poder establecer links vínculos y conectores digitales que permitan ser parte de la experiencia digital del consumidor. (Eouzan, 2013).

Apareció el marketing de contenidos considerando que lo que vende hoy en día es la gestión de contenidos, haciendo referencia que la oferta digital de productos y servicios ya no es única, sino que se ha saturado. (Pedros Piñón, y otros, 2012)

El término SEO en la actualidad también ha diversificado su enfoque, sabiendo que significa Search Engine Optimization, este término no tiene costo alguno, pero al estar directamente 
relacionado con la posición de resultados de los buscadores, es indispensable, hacer planes, gestión y consultorías millonarias en SEO creando un nuevo ámbito empresarial. (Paredes, y otros, 2015)

La clave del internet es lograr que el internauta encuentre el lugar de ahí ya es tarea del marketing de contenidos (Sancho, 2011). La industria hotelera es una de los campos más allegados a la web, donde la caracterización del mercado se ha dado a nivel digital donde plataformas especializadas filtran los hoteles de acuerdo a factores como comodidad, precio, cercanía, etc. (Mendes, 2013)

Con el tiempo la industria turística ha sufrido una expansión llegándose a relacionar y hasta diversificar en temas de transporte, valor agregado y calidad (RODRIGUEZ J.; 2012). Es una de las industrias de mayor crecimiento en el país, datos evidenciados entre 2011 y 2016. La tasa de crecimiento en el periodo de estudio es de aproximadamente 7,84\%, refiriéndose únicamente al crecimiento de turistas, indicando que el gasto promedio anual per cápita asciende a $1000 \$$, definiendo al sector y al mercado como un referencial de un desarrollo muy grande y de oportunidad de inversión grande.(MINTUR; 2015).

Se entiende que la relación entre el marketing digital y el turismo se han interrelacionado de manera que esta industria con el tiempo tiene mayor alcance y ha llegado a representar uno de los pilares fundamentales en el país. La referencia indica que existen al menos treinta mil turistas entendiendo que están segmentados al $50 \%$ en categorías populares y elite en hospedaje y servicios turísticos. Sin embargo, aún no se ha podido cuantificar el monto que representa el hospedaje informal, que al menos llegan al 20\%. (Castellanos, 2013)

El sector en si es un sector que ha crecido de manera exponencial con la relación con el marketing digital, en los últimos años, este crecimiento ha llegado a estandarizarse cerca del $25 \%$, los cuales han colaborado en un crecimiento del $2 \%$ en empleos. Con el tiempo a la par de infraestructura, la calidad de servicio ha crecido entendiendo que el mundo web ha metido presión al exponer a la industria hotelera ecuatoriana con las demás en el mundo. (MINTUR; 2015). 


\section{Materiales y métodos}

El mkt digital es una tendencia post modernista que representa la percepción virtual de un internauta que con el tiempo valora más la realidad a nivel virtual que una publicidad física en decadencia. El proyecto de Investigación actual es de naturaleza explicativa inferencial ejecutado en un año, a manera de una caracterización de segundo nivel. El muestreo se enmarco con un diseño transversal medido varias veces y posteriormente estandarizado con una media, la validez de investigación se centra en la realización de método de expertos y una prueba piloto de depuración. Las variables fueron implementadas a través de preguntas en dos herramientas diferentes en dos enfoques distintos.

\section{Resultados}

\section{Caracterización de la Oferta}
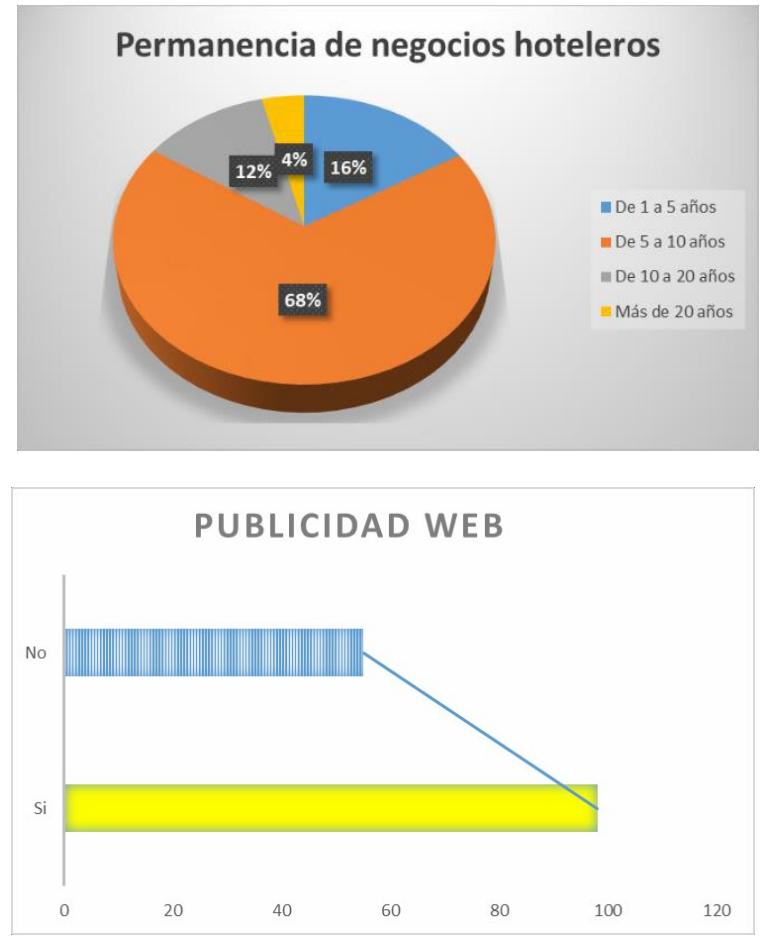

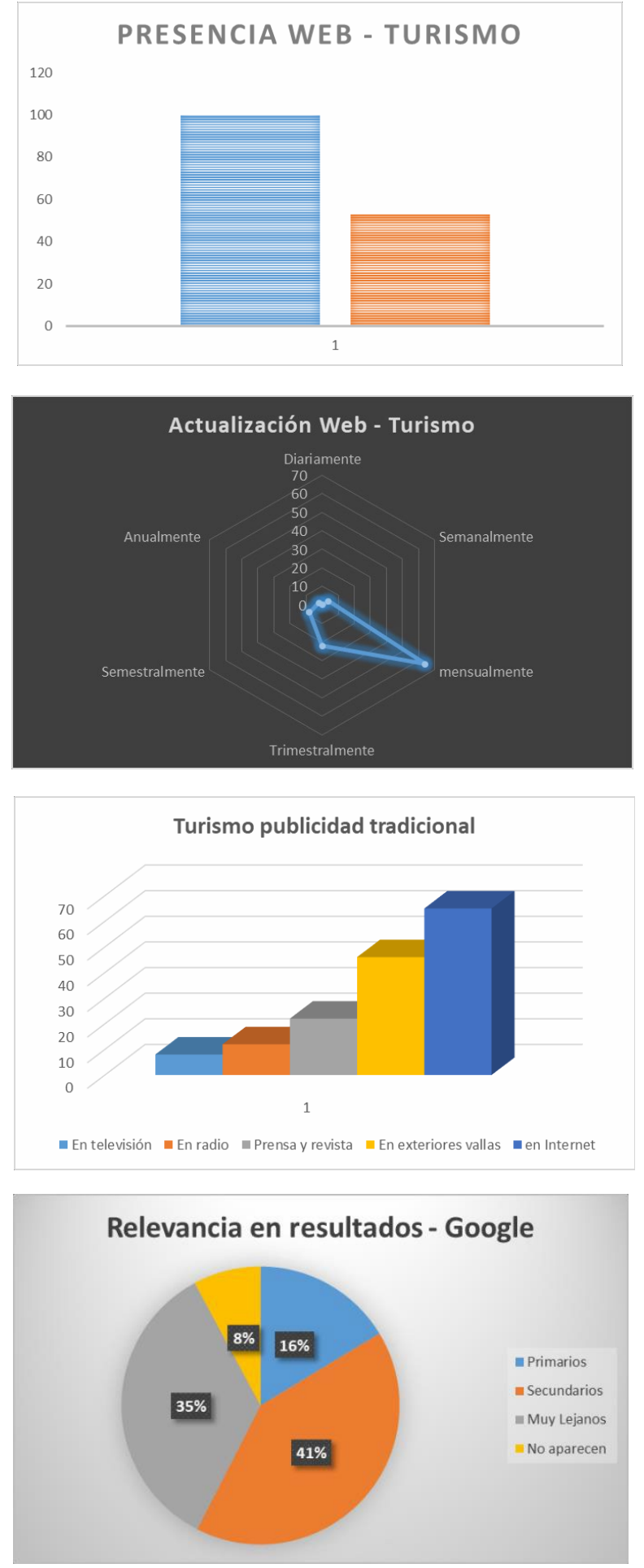

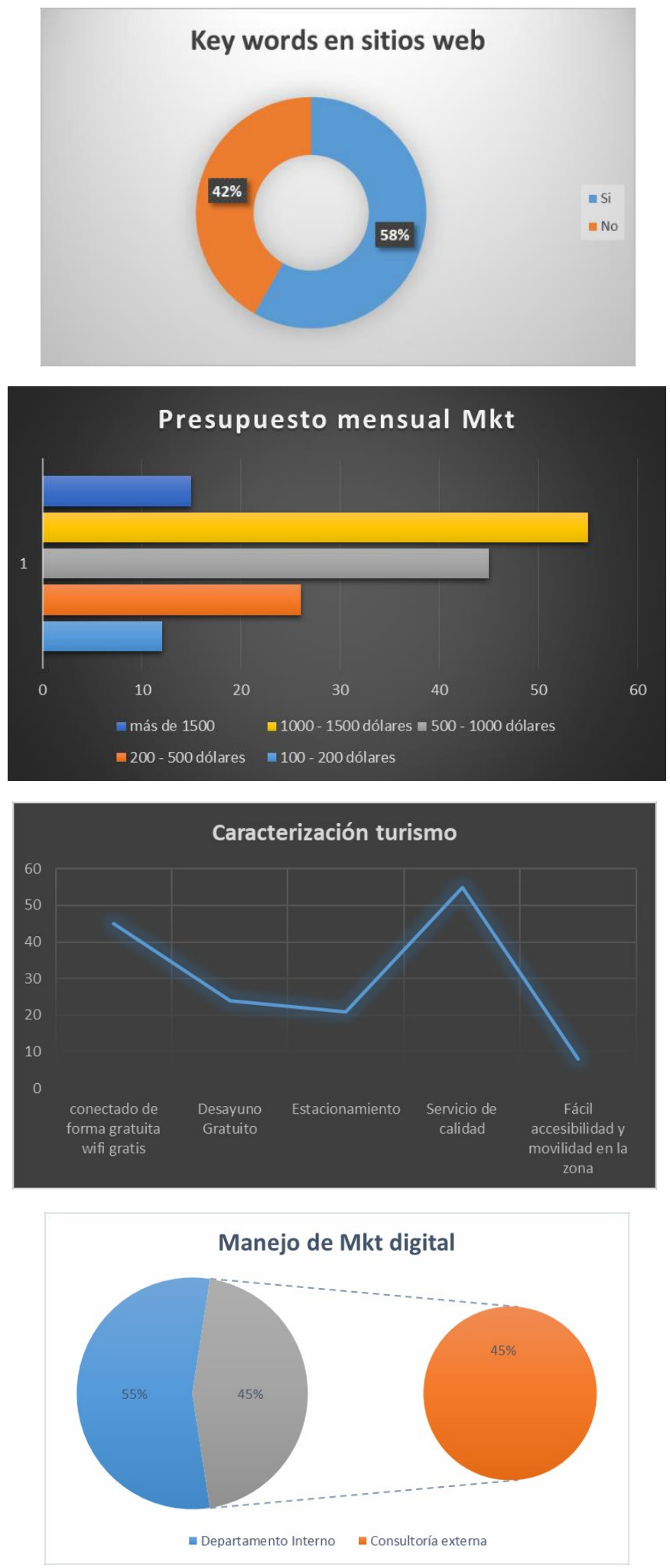

Fuente: Elaboración Propia 


\section{Caracterización de la Demanda}
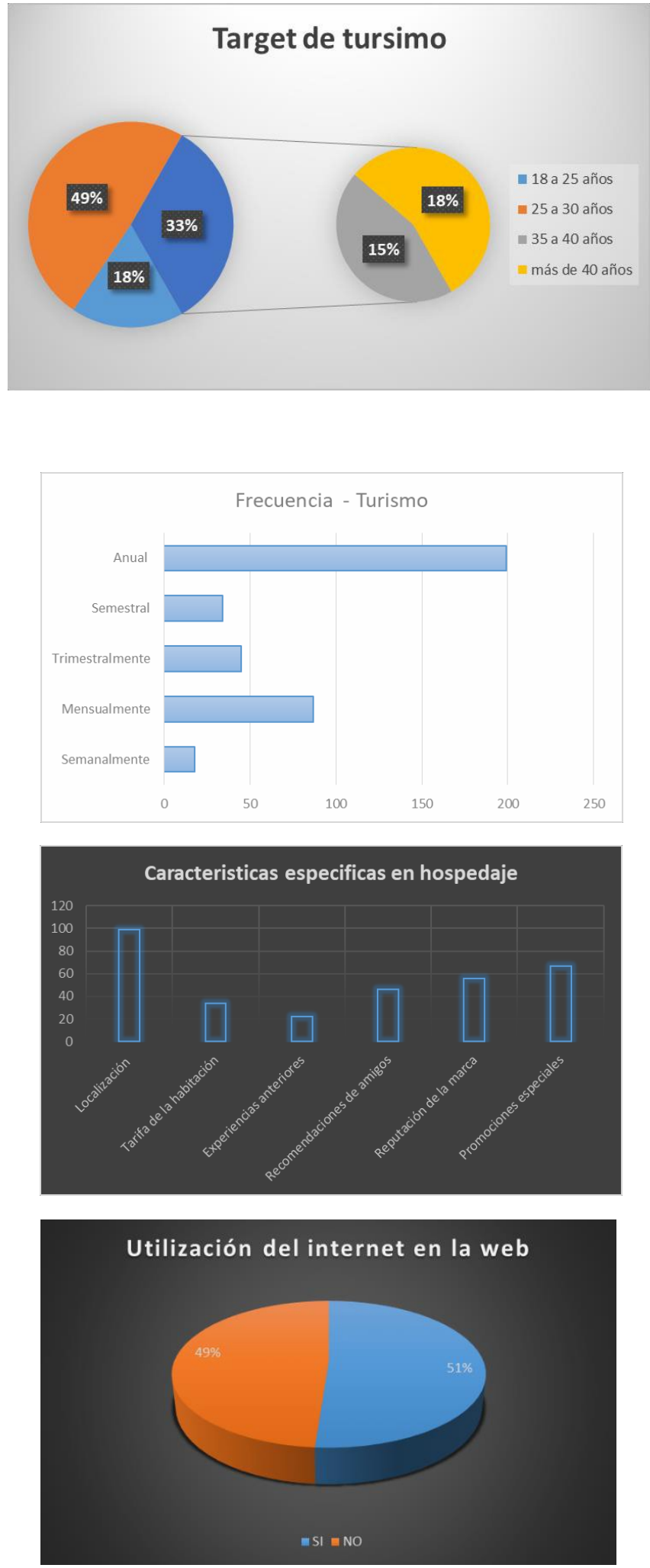

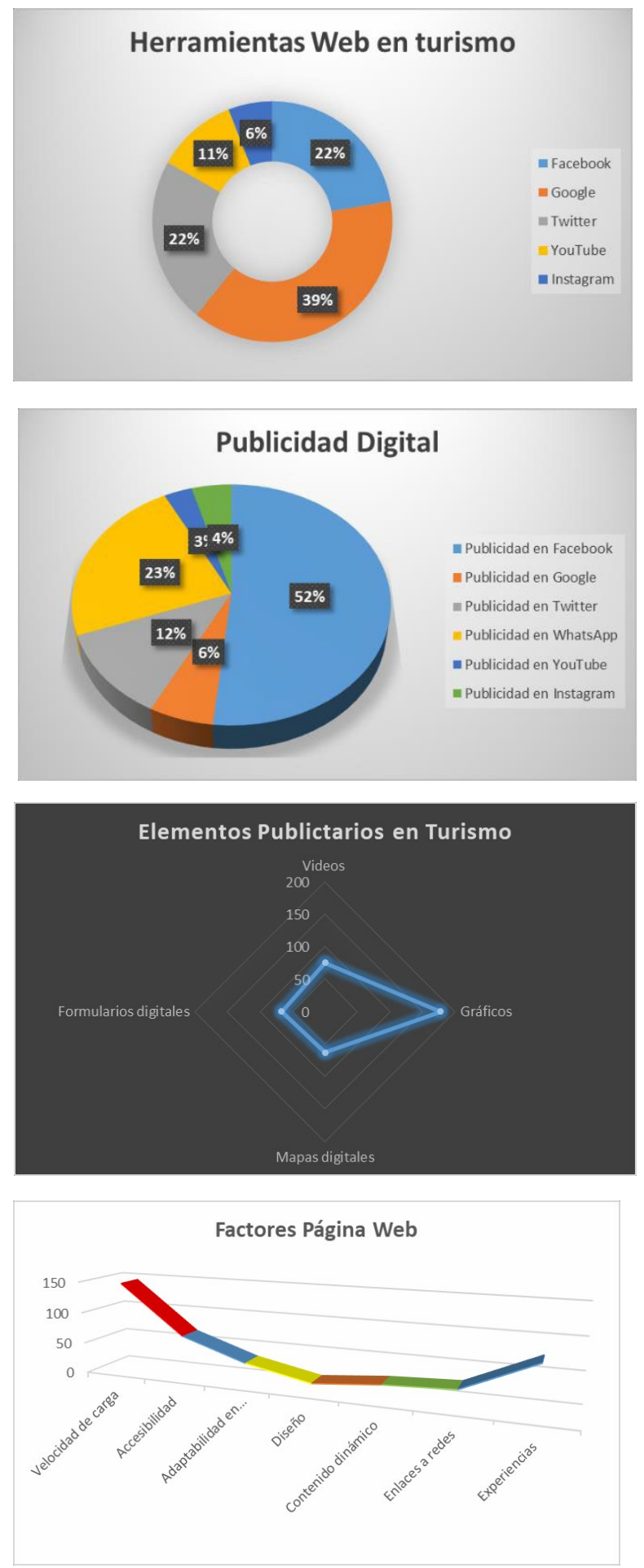

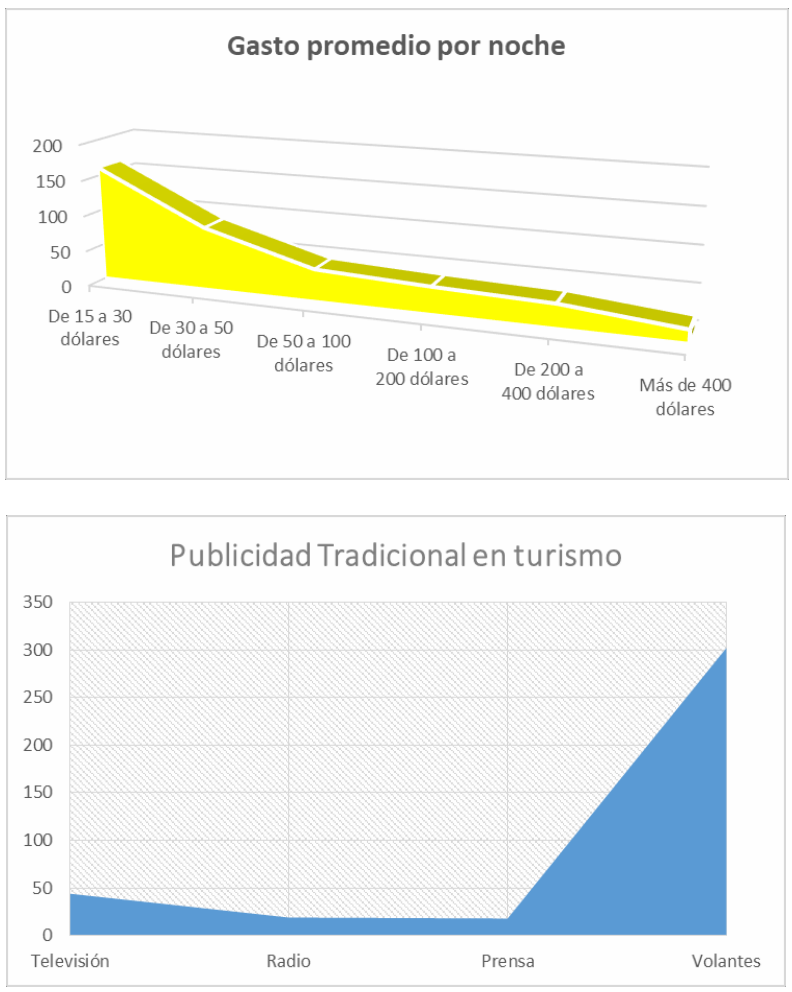

Fuente: Elaboración Propia

\section{Resultados}

H1: Las estrategias de marketing digital SI influye en el posicionamiento SEO en el sector Hotelero en la provincia de Tungurahua

H0: Las estrategias de marketing digital NO influyen en el posicionamiento SEO en el sector Hotelero en la provincia de Tungurahua

\section{Frecuencias Observadas y Frecuencias Esperadas.}

Tabla 2. Frecuencia Observada

\begin{tabular}{llcc}
\hline \multicolumn{1}{c}{ PREGUNTAS } & \multicolumn{2}{c}{ CATEGORÍAS } & SUBTOTAL \\
& Si & No & 383 \\
\hline $\begin{array}{l}\text { Suele reservar o contratar } \\
\text { hoteles en línea }\end{array}$ & 150 & 233 & 383 \\
$\begin{array}{l}\text { Suele utilizar internet para } \\
\text { buscar información sobre }\end{array}$ & 196 & 187 & \\
hoteles y turismo & & & 766 \\
\hline TOTAL & 346 & 420 & \\
\hline FOTAL & &
\end{tabular}

Fuente: Elaboración Propia 
Tabla 3 Frecuencia esperada

\begin{tabular}{lccc}
\multicolumn{1}{c}{ PREGUNTAS } & \multicolumn{2}{c}{ CATEGORÍAS } & SUBTOTAL \\
& Si & No & \\
\hline $\begin{array}{l}\text { Suele reservar o contratar } \\
\text { hoteles en línea }\end{array}$ & 173 & 210 & 383 \\
$\begin{array}{l}\text { Suele utilizar internet para } \\
\text { buscar información sobre } \\
\text { hoteles y turismo }\end{array}$ & 173 & 210 & \\
\hline TOTAL & $\mathbf{3 4 6}$ & $\mathbf{4 2 0}$ & 766 \\
\hline
\end{tabular}

Fuente: Elaboración Propia

\section{Modelo Matemático}

En el modelo matemático se recurrió a la herramienta estadística denominado Chi Cuadrado, considerándose la más apropiada y efectiva para tratar datos cualitativos, utilizándose en esta forma los siguientes elementos:

En donde:

$$
\mathrm{X}^{2}=\sum \frac{(\mathrm{O}-\mathrm{E})^{2}}{\mathrm{E}}
$$

$\mathrm{X}^{2}=$ Chi Cuadrado.

$\sum=$ Sumatoria de.

$\mathrm{O}=$ Frecuencia Observada (Encuestas)

$\mathrm{E}=$ Frecuencia Esperada (Observación)

Nivel de significancia

Sobre el nivel de significancia a implementarse en la presente investigación, se tomó a consideración el valor de 0,05

\section{Cálculo de grados de libertad}

Zona de aceptación y rechazo

Grados de libertad

$$
(\mathrm{G} 1)=(\mathrm{F}-1)(\mathrm{C}-1)
$$

Dónde:

$\mathrm{gl}=$ Grados de Libertad

$\mathrm{C}=$ Columnas de la tabla

$\mathrm{F}=$ Filas de la tabla

Remplazando los datos se obtiene:

Grados de Libertad $(\mathrm{gl})=($ Filas-1) $($ Columnas-1 $)$ 
$(\mathrm{gl})=(\mathrm{F}-1)(\mathrm{C}-1)$

$(\mathrm{gl})=(2-1)(2-1)$

$(\mathrm{gl})=1 * 1$

$(\mathrm{gl})=1$

En consecuencia, se obtiene que el valor tabulado de $(\mathrm{Xt})$ con 1 grados de libertad y un nivel de significación de 0,05 , se encuentra en (3.84)

Tabla 4. Tabla de Contingencia

\begin{tabular}{llccll}
\hline OBSERVADA & ESPERADA & $(\mathbf{O}-\mathbf{E})$ & $(\mathbf{O}-\mathbf{E})^{\mathbf{2}}$ & & /E \\
\hline 150 & 173 & -23 & 529 & 3.06 & \\
233 & 210 & 23 & 529 & 2.52 & \\
196 & 173 & 23 & 529 & 3.06 & \\
187 & 210 & -23 & 529 & 2.52 & \\
766 & 766 & & & 11.16 & \\
\hline
\end{tabular}

Fuente: Elaboración Propia

\section{Decisión Final}

En cuanto a los resultados que se obtuvo sobre el cálculo del Chi Cuadrado Calculado y el Chi Cuadrado Tabulado, se puede aseverar que Chi $2 \mathrm{c}=11.16$ y es $>$ Chi $2 \mathrm{t}=3.84$; en esta forma se acepta la hipótesis alternativa y se rechaza la nula, por lo que se puede decir que:

Hi: Las estrategias de marketing digital SI influye en el posicionamiento SEO en el sector Hotelero en la provincia de Tungurahua

\section{Conclusiones}

El 70\% de los establecimientos turísticos en la región tienen una madurez y experiencia importantes con un promedio de 7 años en el negocio, considerando que la sinergia de su permanencia debe haber creado un conocimiento implícito grande.

El 40\% de las empresas aun no manejan publicidad ideológica web, sin embargo su presencia a nivel digital tiene una tendencia creciente con páginas web en plataformas estándar.

La industria, al ser mayormente gráfica maneja un gasto mensual en publicidad entre física y digital de 1000 a 1500 dólares, de los cuales gran parte está distribuida en vallas publicitarias, entendiendo que al inteligenciarse en la parte digital este rubro podría ser reinvertido en otros puntales de desarrollo.

Se puede evidenciar que las páginas web están medianamente desatendidas considerando que su actualización se realiza únicamente de forma mensual sin considerar la alta rotación y actualización del ciberespacio. 
En la industria se valora mucho por el cliente, representando el factor más importante el servicio de calidad y dentro de este la conexión con el mundo virtual a través del wifi, en el mercado las empresas prefieren dejar el manejo de redes y la gestión web en general en un $45 \%$ a especialistas o Community manager.

La gestión digital en la zona es pobre considerando que el posicionamiento de las empresas de turismo en los buscadores solo son primarias en un 16\%, indicando una deficiente gestión de contenidos y keywords, estas últimas solo son manejadas por el $56 \%$ de la oferta.

El perfil del turista se forja desde los 18 años donde la percepción, ilusión y motivación del mismo se potencia hasta los treinta y posteriormente a esto su percepción puede ser modificada en menor proporción.

La forma de llegar al cliente en esta industria se da por material gráfico y material audiovisual, entendiendo que la mayoría de personas tienen un sesgo de percepción por este tipo de medios. Al momento de elegir un hotel se hace referencia a la localización del mismo, siendo un factor Correlacional de este y por otra parte a promociones, indicando que el target latinoamericano se maneja mucho por este.

El turista al momento de visitar una página de turismo hace referencia a la velocidad de carga de la misma y a la UX o User Experience donde indica que no solo debe ser funcional sino dar valor agregado.

Los targets populares son aquellos que suelen tener masificación, sin embargo el medio que más llega a nivel de turismo considerando el entorno virtual es el de Facebook.

\section{Referencis Bibliograficas}

Adolpho, C. (2011). Os 8 Ps do Marketing Digital: O guia estratégico de marketing digital. Novatec Editora.

Andrade, David Alveiro. 2012. Estrategias de Marketing Digital. Maracaibo: s.n., 2012.

Bernal, Cesar. 2010. Metodología de la Investigación. 2010.

Brinkley, C. (2012). Marketing Digital. Editex.

Brodkin, J. (2015). Gartner touts Web 2.0, scoffs at sequel. Network World.

Burgos, E. (2009). Del 1.0 al 2.0: Claves para entender el nuevo marketing.

Cangas Muxica , J. P., \& Guzmán Pinto , M. (2010). Marketing Digital: Tendencias en su apoyo al E-commerce $\mathrm{y}$ sugerencias de implementaciòn. Retrieved from 
http://repositorio.uchile.cl/tesis/uchile/2010/ec-cangas_jp/pdfAmont/ec-cangas_jp.pdf

Cárdenas, Johana y Ponce, Ingrid. 2013. Estudio de Indicadores que permitan el Posicionamiento del Website de la Universidad Politécnica Salesiana. Guayaquil: s.n., 2013.

Castellanos, L. (2013). El turismo en un Ecuador megadiverso. Revista gestión, 46.

Chaffey, D., \& Ellis-Chadwick, F. (2012). Digital marketing: strategy, implementation and practice (Vol. 5). Harlow: Pearson.

Chakravorti, B. (2010). Stakeholder Marketing 2.0. Journal of public policy \& marketing, 29(1), 97-102.

Clark, D. (2012). Digital Marketing. España: McGraw-Hill Interamericana.

Consoli, D., \& Musso, F. (2010). Marketing 2.0: A new marketing strategy.

de Vicuña Ancín, J. M. S. (2018). El plan de marketing digital en la práctica. Esic editorial.

Empresa Metropolitana Publica Quito; Cifras llegadas de turistas al DMQ, 2013

Eouzan, Guillaume . 2013. Marketing web: Definir, implementar y optimizar nuestra estrategia

2.0. Barcelona : ENI, 2013.

Equipo Vértice. (2007). Marketing promocional orientado al comercio. Editorial Vértice.

Erragcha, N., \& Romdhane, R. (2014). New faces of marketing in the era of the web: from marketing 1.0 to marketing 3.0. Journal of Research in Marketing, 2(2), 137-142.

Espinoza. 2014. Marketing Estrategico. 2014.

Galarza, Antonio. 2003. Tipos de Investigación. Chile: s.n., 2003.

González Rodrigo, E., Sainz González, J., \& Torrens Sellens, J. EL TURISMO EN EL ENTORNO RURAL. USO DE LAS NUEVAS TECNOLOGÍAS PARA ACCEDER AL MISMO.

González, J. A. M. (2011). MARKETING TURÍSTICO ONLINE.TURyDES, 4(9).

Gutiérrez, J. C. (2008). El libro del Marketing Interactivo y la Publicidad digital. España: ESIC.

Hanson, W. A., \& Kalyanam, K. (2000). Principles of Internet marketing. Cincinnati: SouthWestern College Pub.

Herrera, Luis, Medina, Arnaldo y Naranjo Galo. 2004. Tutoría de la Investigación Científica. Quito: s.n., 2004.

Iglesias, M. (2013). El Marketing Digital en las licenciaturas en administración de argentina.

Retrieved from http://bdigital.uncu.edu.ar/objetos_digitales/5560/iglesias-tesiscsec.pdf

Kotler, P. (2004). Forum Mundial de Marketing y Ventas,. Barcelona España. 
Kotler, P. (2013). Marketing Internacional. España: McGraw-Hill/Interamericana.

Kurzwell , R. (2000). The Age of Spiritual Machines - When Computers Exceed Humanuman Inteligence. Nueva York: Pinguin Putman inc.

Lluis, Codina y Marcos, Mari Carmen. 2005. Posicionamiento Web. Venezuela : s.n., 2005.

Lozano, M. J. F., \& Blázquez, A. T. (1999). La incorporación de la microsegmentación en los planes de marketing y expansión de las franquicias. Investigación y marketing, (64), 1119.

Matthew, K. (2013). Making digital and traditional marketing work together. Paraninfo.

Medina, I. G. (2011). Marketing digital multimedia: Nuevos formatos y tendencias. Revista Géminis, 2(2), 37-45.

Mendes Thomaz, G., Biz, A. A., \& Gândara, J. M. G. (2013). Innovación en la promoción turística en medios y redes sociales: Un estudio comparativo entre destinos turísticos. Estudios y perspectivas en turismo, 22(1), 102-119.

Ministerio de Turismo, Rendición de Cuentas 2016

MINTUR, M. D. (2015). PLAN ESTRATÉGICO DE DESARROLLO DE TURISMO SOSTENIBLE PARA ECUADOR “PLANDETUR 2020”. Recuperado de: http://www. turismo. gob. ec/wpcontent/uploads/downloads/2013/02/PLAN DETUR-2020. pdf.

Moschini, S. (2012). Claves del marketing digital. La nueva comunicación empresarial en el mundo, 3 .

Nuñez, A. (2010). Marketing Digital. España: Editorial Vértice.

Paredes, Jéssica y Maridueña, Andrés. 2015. Plan de Marketing Digital para la empresa Corporación de Servicios TBL de la Ciudad de Guayaquil . Guayaquil : s.n., 2015.

Pedrós Piñón, María, Arrabal, Gonzalo y Panzano, José. 2012. Manual SEO. Posicionamiento web en Google para un marketing más eficaz. S.1. : Bubok, 2012.

Pérez, E. D. (2001). Sobre modas y realidades: CRM o el nuevo marketing digital. Nueva Econ. y Empres., vol. Abril-Mayo, (791), 55-62.

PROECUADOR, Perfil Turístico 2016.

RODRIGUEZ VALDIVIESO JESSICA; "Análisis De La Aplicación Del Marketing En Establecimientos De Alojamiento De Lujo Y Primera En La Ciudad De Cuenca (20072009); CUENCA 2012.

Rowland, C., Goodman, E., Charlier, M., Light, A., \& Lui, A. (2015). Designing connected 
products: UX for the consumer internet of things. " O'Reilly Media, Inc.".

Ryan, D. (2016).Understanding digital marketing: marketing strategies for engaging the digital generation. Kogan Page Publishers.

Serrano, M. (2016). Marketing Digital. México: McGraw-Hill.

Sevilla, P. (2008). marketing digital. México: primera.

Tuten, T. L. (2008).Advertising 2.0: social media marketing in a web 2.0 world: social media marketing in a web 2.0 world. ABC-CLIO.

Ugarte Otero, Xoán. 2011. Imagen y posicionamiento de Galicia como destino turístico a nivel nacional e internacional. S.1.: Univ. Santiago de Compostela, 2011 